\title{
DESIGN OF AN OBJECT IDENTIFICATION SYSTEM WITH A THERMAL CAMERA
}

\author{
Mario Karpuzi \\ Dept. of Industrial Design \\ and Production Engineering, \\ University of West Attica \\ Athens, Greece
}

\author{
Konstantinos Georgiou \\ Dept. of Industrial Design \\ and Production Engineering, \\ University of West Attica \\ Athens, Greece
}

\author{
Michail Papoutsidakis \\ Dept. of Industrial Design and \\ Production Engineering, \\ University of West Attica \\ Athens, Greece
}

\author{
Avraam Chatzopoulos \\ Dept. of Industrial Design \\ and Production \\ Engineering, \\ University of West Attica \\ Athens, Greece
}

Abstract - In this paper we will present and cover the study of particular tools and devices where they will be used to implement an automated object separation system. In a nutshell, it will be a system where it will separate, depending on our needs from the objects we place on its original position. More detailed, the basic tools for implementing this automation are divided into three categories. The first category will be the basis of our system the second will be the sensors and finally the third the controller and the power supply. The base of the system will consist of a motor, two rollers, a conveyor belt and a gear unit where it will control the engine speed. The sensors that will be used will be a thermal camera and a laser, where they will be located in the center of the conveyor belt with the thermal camera directly above the laser. Also our controller will be a PLC where it will give us more flexibility in the programming of the thermocamera. More specifically, in this thesis we will test bottles, i.e. by placing a bottle in our original position where it will be the center of the conveyor belt, the laser sensor will signal to activate the camera. As soon as the thermocamera is activated, the programmable analogue of each user will give the PLC information to approve or discard the bottle at its level. Then you send this information as a command to the engine to act clockwise or counterclockwise, i.e. to send the bottle to the stack with those approved or those that have been discarded. Finally, the system will be powered by PPC, but there will be a choice and usage of a photovoltaic that saves energy and makes our construction more environmentally friendly.

\section{Keywords - Watermarking, Haar Wavelet, DWT, PSNR}

\section{INTRODUCTION}

In this chapter we will see general information on the materials to be used for the implementation of our automated system. These materials are rollers, conveyor belts, gear motor, sensors, controller and photovoltaic.
Starting from the roller is a mechanical device used to change the direction, to drive and stretch the belt in a conveyor system and is one of the most important components in conveyor belts. When someone uses the right accessories of this kind, it is certain that the conveyor belt will work the way it was designed. Correctly manufactured rollers, both in terms of materials and quality of construction, ensure the conveyor belt has a lifetime and maximum performance.

The conveyor belt is the conveying means of a conveyor system consisting of two or more pulleys, when they start to rotate the belt also starts to rotate with them. One or both pulleys are fed by moving the belt and the material. The feed pulley is called the drive pulley while the pulley without motor is called the idler pulley. Belt conveyors are generally quite similar in their construction, consisting of a metal frame with rollers at each end of a flat metal bed. The belt rotates around each of the rollers and when one of the cylinders is fed by an electric motor, the belt slides along the solid metal bed, moving the product. In heavy-duty applications, cylinders are required. The cylinders allow weight transfer as they reduce the amount of friction produced by the greatest load on the belt. Belt conveyors are the most commonly used conveyors because they are the most versatile and less expensive. The product is transported directly to the belt so that both normal and irregular objects, large or small, light and heavy, can be transported. These carriers should only use high quality products, which reduce belt stretching and lead to less maintenance for voltage adjustments. Belt conveyors can be used to transport the product in a straight line or through a change in elevation or direction. In some applications they can also be used for static accumulation or cartons.

As for the engine, a DC motor will be used in our system. DC motors first appeared in the late 19th century. Despite the development of alternating current systems (AC) was high, the DC motors continued to be present. One main reason is that we find them in applications that make great changes in the speed of rotation. DC motors are in the category of rotating electric motors, where their main function is the conversion of 


\section{International Journal of Engineering Applied Sciences and Technology, 2019 \\ Vol. 4, Issue 5, ISSN No. 2455-2143, Pages 471-477 \\ Published Online September 2019 in IJEAST (http://www.ijeast.com)}

electrical energy into mechanical energy. Their function is based on electromagnetic induction, that is, when a conductor moves into a magnetic field, an electrical potential is generated within the conductor, resulting in the occurrence of electrical current in the conductor. When a conductor running through an electric current is found in a magnetic field, you are exercising a force from the magnet field that tends to move it. This power is proportional to the intensity of the electric current, the intensity of the magnetic field, but also the length of the conductor. Transmission control of rotary drive and transmission of power from drive to driven rotary shaft require gearboxes. They are machines that are mounted on the motor and can reduce the speed that the motor provides, depending on the transformation ratio they have. Typically, motors are built with the gearbox, especially in small DC motors.

Technology has advanced so that in our everyday life we can find sensors almost everywhere their main purpose are to help and facilitate the life of the man. The sensors give information about state changes such as position, speed, power, movement, temperature, pressure, capacity, level, and much more. Special sensors can also detect chemical quantities, radiation, and more. Sensors can be found as individual devices or even constructions, but this does not alter their basic function, which is no other than detecting a signal or excitation and converting it into a measurable output where it helps the user, i.e. voltage or current.

The most important part in an automated system is the controller. In our system a PLC (Programmable Logic Controller) will be used. The PLC is a microprocessor-based controller and embedded reprogrammable memory. Commands are stored in the memory that is running during operation. The PLC first appeared in the 1970s, the difference with other controllers was the storage of a program in its memory, a great advance of the era. Then for data compatibility and data exchange, efforts were made to require communication protocols as well as to create software so that users can even program a calculated one. Nowadays, PLCs find great application because of their capabilities

By the term photovoltaic we declare the industrial arrangement of many photovoltaic cells in series, more detailed are semiconductors crafted to form an electric circuit in series. The purpose of photovoltaic is to take solar radiation and turn it into electric voltage, this is done with semiconductors where photons are absorbed by the solar radiation and generate voltage. We call this phenomenon Photovoltaic effect. The first human acquaintance with the photovoltaic effect occurred in 1839 when the French physicist Edmond Becquerel discovered the photovoltaic phenomenon during his experiments with an electrolytic contact made of two metal electrodes. The growth of photovoltaic was very large, mainly between 1992 and 2017, and continues. However, in the meantime, photovoltaic have evolved from a specialized application market to a major source of electricity.

\section{PARTS OF OUR CONSTRUCTION}

\section{A. Rollers}

The right choice of rollers is to do with the economic evaluation of the offered materials. Therefore, in our market, we must pay attention to the following:

- The purchase cost

- $\quad$ The operating costs, i.e. installation, maintenance and consumption

- The cost of replacing it in case of damage or disfunction

In our system it is imperative to use two rollers that will be placed at the ends of the conveyor belt. The rollers are used for the correct movement of the tape but also for its alignment, by saying alignment we put the correct position so that the tape is stretched and does not leave its position. The DC motor will only drive one of the two rollers that will move the tape, where it will in turn rotate the other roller. The rollers that will serve us for the conveyor belt are two of the following types:

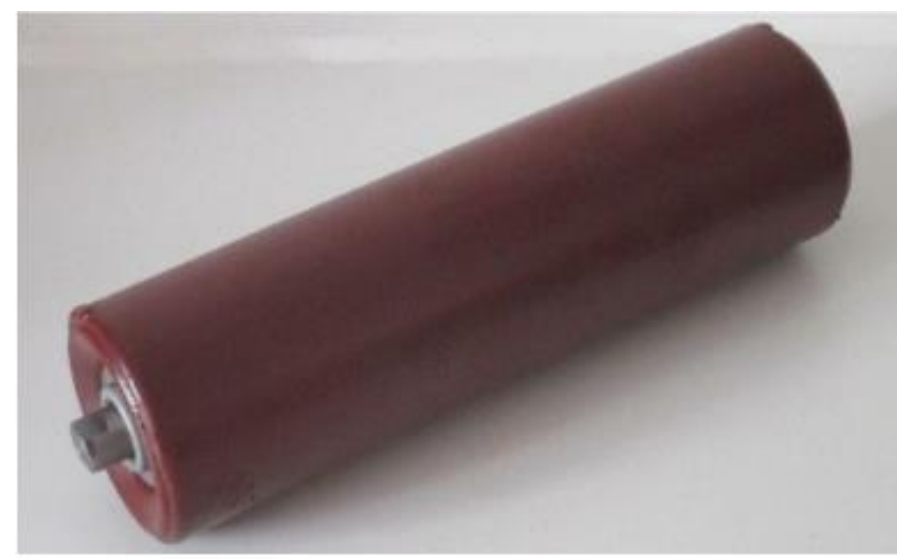

Fig. 1. Markenlos Roller

This roller is from Markenlos, of Polish origin, with a roll length of $25 \mathrm{~cm}$ and a total length of $27,6 \mathrm{~cm}$. It is made of steel with a total diameter of $8.9 \mathrm{~cm}$ and a diameter of its axes at $2 \mathrm{~cm}$. Also we could use if we also want roller coat sleeves where they are used in a wide range of industries. The sleeves also provide a soft and smooth surface while protecting the product from wear and tear.

\section{B. Conveyor Belt}

A conveyor belt consists of two pulleys with a continuous loop of material and the belt rotatable thereon. The drive pulley is called the pulley being fed while the other is called an intermediate drive wheel. The belts usually consist of two layers of material, one of which is the lower layer of material used to provide the linear force and shape, which is called a carcase and a layer of surplus to cover it. The carcase consists 


\section{International Journal of Engineering Applied Sciences and Technology, 2019 \\ Vol. 4, Issue 5, ISSN No. 2455-2143, Pages 471-477 \\ Published Online September 2019 in IJEAST (http://www.ijeast.com)}

of a cotton or plastic cloth or mesh and the surplus layer consists of rubber or plastic compounds that are dependent on the use of the belt.

In our system we will need a conveyor belt to move the bottles, i.e. the conveyor belt will be the base where the bottles will be discarded or accepted. A suitable type of tape, where it will be applied on the rollers, is that of the Volta manufacturer and more specifically the FZ type with a 95A / 46D hardness, i.e. a strip made of soft and flexible material with a thickness of $5 \mathrm{~mm}$. It also has a coefficient of friction in steel of 0.36 and has a tolerance of temperatures from $-30^{\circ} \mathrm{C}$ to $60^{\circ} \mathrm{C}$. Having a minimum pulley diameter of $8 \mathrm{~cm}$ is suitable and applies to the rollers selected with a diameter of $8.9 \mathrm{~cm}$. The length of the tape we will need for the implementation of our app will be $148 \mathrm{~cm}$, because we want the treadmill to move the production bottles to $60 \mathrm{~cm}$ while its width will be $20 \mathrm{~cm}$, i.e. $5 \mathrm{~cm}$ less than the length of the rollers will be used.

\section{DC Motors}

Electric motors are used to drive an unlimited range of gears, covering a huge range of applications. In particular, DC motors offer great comfort in reliably controlling movements in industrial mechanisms that are actuated by them because of the great ease in controlling their axle speed. Their main advantage also in relation to AC motors is that for a given power they can develop significantly more mechanical torque on their shaft, making them the most suitable for controlling movements in industrial mechanisms where they need to handle significant mechanical loads.

When we want to use an engine for one of our applications, it must meet some specifications to suit the functional requirements of the mechanism or machine it will move. These specifications have to do with:

- The feed system

- The conditions of the specific area of the field

- The requirements of the electrical power that should be offered to the energized machine and the mechanical power that it should deliver

- Other functional features

Engine components and how to connect the cables of the external power source to it

An important part of our system is the movement of the conveyor belt. Consequently, a DC motor will be used to drive the conveyor belt. DC motors are suitable for such structures as ours. The engine of our choice will be placed at one end of the conveyor belt to drive one of the two rollers installed, where this will in turn drive the whole belt.

The key criterion of the engine chosen will be to be able to move the film with the load it will bring, in our case the bottles. In the conveyor belt you will put a bottle at a time, so its weight will not exceed two pounds. An engine that can handle our construction is Premium Planetary Gear Engine 313 RPM HD.

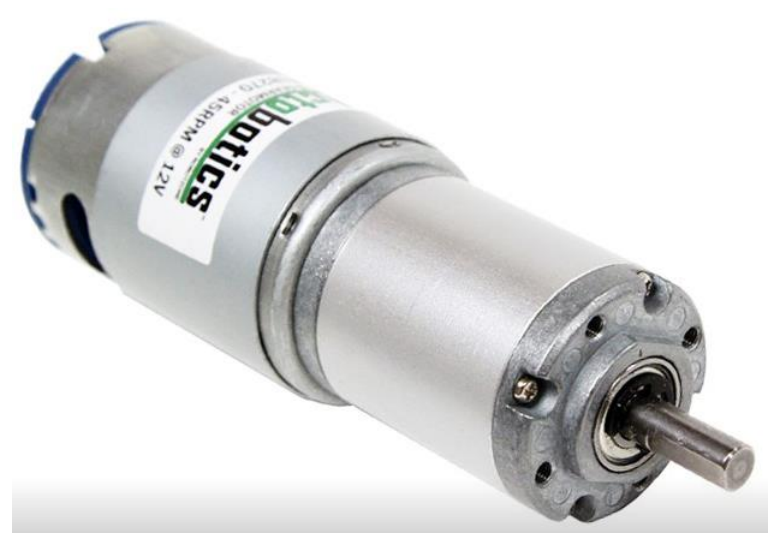

Fig. 2. Premium Planetary Gear Motor 313 RPM HD

This particular engine is made by Actobotics from the US. It consists of metal with a weight of $0.73 \mathrm{kgs}$. It has a voltage range of $6 \mathrm{~V}$ to $12 \mathrm{~V}$ and current $20 \mathrm{~A}$. It also rotates at a speed of $313 \mathrm{rpm}$ and its torque is $135 \mathrm{~kg} * \mathrm{~cm}$.

As for gear units that help us control the transmission of the rotary motion of our engine, we usually find them together with the engine we want to use, especially in small DC motors wherever we use our construction. So the gear unit we use in our system is built into the engine we chose with a 27: 1 transmission ratio.

\section{Sensors}

For the proper sensor selection, a basic parameter is the use it will have, that is, it could be for industrial use where the sensor should be as accurate as possible so there are no errors that can destroy an entire production. The exact choice of a sensor also depends on the nature of the parameters to be measured and other factors, such as the reliability, cost and quality of the information required for the sensor. Another important parameter is the space where the sensor will be placed, for example if it is in a place that may be affected by weather conditions then the sensor should be durable and accurate so that there are as few errors as possible from external factors.

In our system we will need a sensor that will check if there is a bottle in our original position. There is a wide variety of sensors, but in our case a laser sensor would be suitable. This is because the lasers produce monochromatic light which propagates in a particular direction by forming narrow beams, so you give us the possibility when the light break line changes the sensor state. We also wanted the sensor not to be affected by external conditions such as daylight. In conclusion, we could use two different ways to implement this process which both end in the same result. 


\section{International Journal of Engineering Applied Sciences and Technology, 2019 \\ Vol. 4, Issue 5, ISSN No. 2455-2143, Pages 471-477 \\ Published Online September 2019 in IJEAST (http://www.ijeast.com)}

1. The first way is to use a laser sensor to measure distance. So when the distance measured by the sensor decreases, it means that you insert a bottle in the initial position of the conveyor belt, so then the heat sensor of the thermal camera is turned on. The laser will be positioned at the side of the initial position $10 \mathrm{~cm}$ from the conveyor belt and under the heater at a height of $5 \mathrm{~cm}$ from the conveyor surface. One such sensor is the SICK Company's DT20 Hi where it measures distances in four scales from $5 \mathrm{~cm}$ to $100 \mathrm{~cm}$ with great precision.

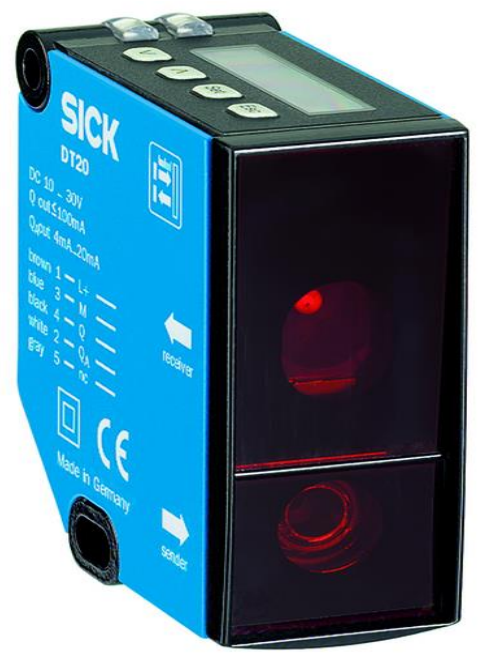

Fig. 3. DT20 Hi Sensor

2. The second way is to use a simple laser where it will be aimed on a photoresistor located on the other side of the conveyor belt just opposite of the laser. In this case, our sensor will be the photoresistor where it will be placed in a black frame to have as little interference as possible from the outside light. So when the light beam of the laser is cut off from the bottle, the photoresistor will change value and as a result the process starts and the camera is switched on.

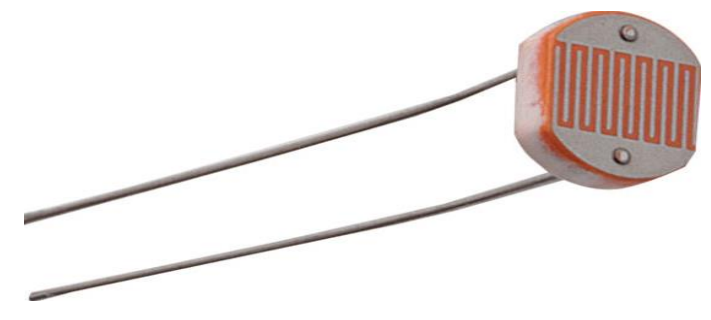

Fig. 4. Photoresistor LDR 5mm

Our automated system will work with both sensor sensors. By using the laser as a sensor, where it is a safer choice, we will have more precision and more options for different uses in the future but increased costs. While using the resistors there are minor errors, mainly due to external factors, but the cost will be greatly reduced.

\section{E. Thermal Cameras}

The camera is a tool that helps us to see the temperature of an object, i.e. it can record the thermal radiation of each object. It is important to know that each object has a thermal radiation even a piece of ice. This method is called thermography. Thermography or infrared photography refers to the thermal radiation detection and thus a visual representation of the thermal signal is obtained. Thermography does not directly measure the temperature of a surface but the change in surface radiation. Thermography is based on the principle that each surface emits energy in the form of temperature radiation. The wavelength emitted depends on the temperature. The photogrammetry is done in a color image where, depending on the different temperatures present, the object we want to control, also corresponds to different colors. Typically blue is referred to as the coolest and red to the warmest.

The Heat Camera uses lenses to gather and focus the radiant heat energy on a sensor. This sensor is sensitive to the energy of the infrared range of the electromagnetic spectrum and converts the detected energy into an electrical signal that is detected, processed and displayed in a colorful, usually, picture where the different temperatures correspond to a color palette and make the target thermal state distinct.

Our system has as its main function the separation of bottles. For this reason, the most important tool for this separation in our study is our basic sensor, which is none other than the camera. The camera should be positioned at a convenient location so that its lens looks at the original position of our system where the bottles will be placed. This way and with the appropriate software uploaded to our controller will separate the bottles from the license. Therefore, it will be placed in the center of the corridor and above the low-sensing laser. A better solution would be on the construction and in particular the base where the hot-plate would be placed was easily movable so that the camera could change its angles and height for later use of this system in other processes than the sorting of bottles. In our case, however, there will be no problem if the camera is stable. A hot camera that meets our requirements for the system to be created is FLIR A615 of the flir company.

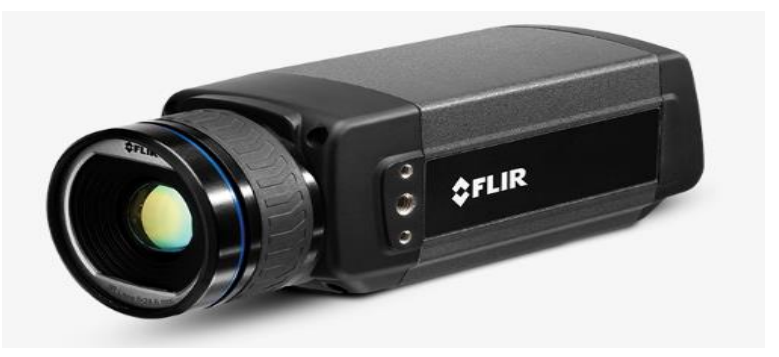

Fig. 5. Thermal Imaging Camera FLIR A615

This hotspot with a resolution of $640 \times 480$ pixels and a $360 \times 180 \times 550 \mathrm{~mm}$ dimension has communication protocols where they are compatible with our controller so they can 


\section{International Journal of Engineering Applied Sciences and Technology, 2019 \\ Vol. 4, Issue 5, ISSN No. 2455-2143, Pages 471-477 \\ Published Online September 2019 in IJEAST (http://www.ijeast.com)}

communicate and exchange data. There is also the ability to connect with a computer for data storage and database creation, where it is important nowadays for more information and studies.

\section{F. Programmed Logic Controllers (PLC)}

The PLC has a sequence of functions performed cyclically. First, it recognizes inputs which it converts to digital form and stores it in the input representation memory. Then the microprocessor processes the data from memory and runs the program, this processed data is stored in the cost representation memory. Finally, output signals are generated by the output representation memory.

The PLC consists of:

1. Power supply unit

2. Central processing unit

- consists of Memory

- $\quad$ and the Microprocessor

3. Inputs and Outputs ( $\mathrm{I} / \mathrm{O})$

4. Digital inputs

5. Digital outputs

6. Analog inputs

7. Analog outputs

8. Real time clock

The main tool in each device is the controller, so in an automated system where there is a sensor with many features such as a thermo camera, the appropriate option is a controller that will give the user the convenience of planning according to his wishes. The PLC is a powerful controller and gives the developer the flexibility to do exactly what he wants without errors and lateness.

In this automation where bottles will be tested no other controller could be used due to the thermal camera where it is a complex sensor and difficult to program. It can also be connected to the laser and the motor without any problems.

A PLC where we can make use of is the Modicon M241:

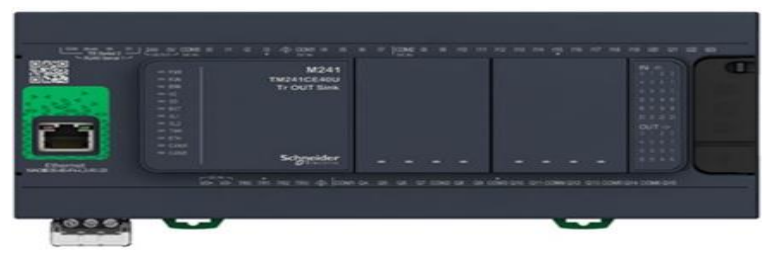

Fig. 6. Logic Controller - Modicon M241

This PLC of Schneider Electric has 24 digital inputs, 16 digital outputs and can be powered by $100 \mathrm{~V}-240 \mathrm{~V}$ AC. It is suitable for our needs and you have a lot of use in automation installations.

\section{G. Photovoltaics}

The photovoltaic system consists of:

- The photovoltaic generator with mechanical support and possibly a solar tracking system.

- Power definition and control device including care for measurement and observation.

- Backup generator.

Our system can also be powered by a photovoltaic panel in order to be environmentally friendly and economical. The panel cells will encapsulate solar energy and store it as a current in the battery where you will be consuming. In order to make the correct panel selection we need to know the total system consumption, so we can choose the dimensions and number of panels we will need. Then you have to choose the battery which, in addition to supplying the system, should be suitable so that it can store energy and re-supply it to our system.

Specifically for our system, we will need a 80Ah deep discharge battery for more comfort, protection and longer battery life. A profiled deep-discharge battery with these specifications is the SOLAR POWER SP110, the INTACT construction that comes from German.

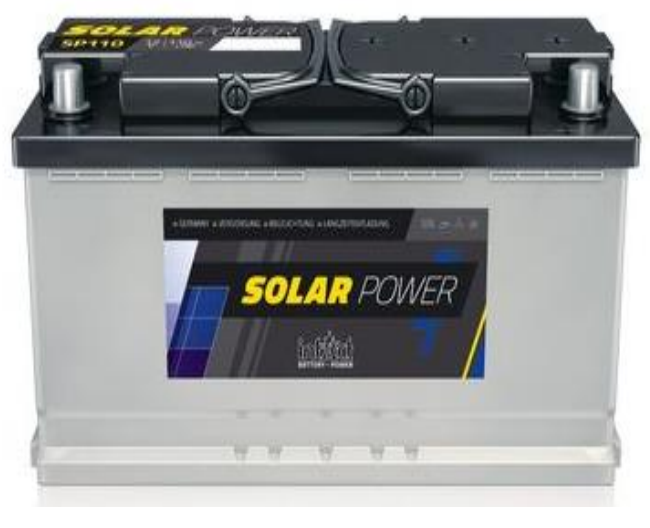

Fig. 7. SOLAR POWER SP110

This battery is made with $110 \mathrm{Ah}$ and $12 \mathrm{~V}$ voltages. It has 800 life cycles at $30 \%$ discharge. It is suitable for stand-alone photovoltaic and electric outboard. It is also open type with liquids and has special separators and reinforced plates with a thickness of $2.6 \mathrm{~mm}$ and $2.2 \mathrm{~mm}$. It has a weight of $24.5 \mathrm{kgs}$ with dimensions:
- $\quad$ Width $=17.5 \mathrm{~cm}$
- $\quad$ Length $=35,3 \mathrm{~cm}$
- $\quad$ Height $=19 \mathrm{~cm}$ 


\section{International Journal of Engineering Applied Sciences and Technology, 2019 \\ Vol. 4, Issue 5, ISSN No. 2455-2143, Pages 471-477 \\ Published Online September 2019 in IJEAST (http://www.ijeast.com)}

As for the photovoltaic panel it should be about 80Wp. Here are many choices in manufacturers and sizes.

As we have predicted, the installation of the photovoltaic system for the autonomy of our system is not insurmountable, because all the construction will be fed from the PPC network. Putting it in an industry where there will be a larger production line will help financially but automatically make you more environmentally friendly.

\section{COST AND FUTURE USE OF THE PROJECT}

A key part of whatever we are trying to build or create is the economic part, especially nowadays. So here we will calculate the cost of our construction. The automated system we have seen above will be built for use primarily on production lines of an industrial plant but could be implemented even by an independent person for his own use, for the implementation of a project or even for studying the operation of the electrical or mechanical of the party. In the table below we will see the values of the tool-kits that will be used in our system.

Table -1 System Materials Prices

\begin{tabular}{|c|c|c|}
\hline $\begin{array}{c}\text { APPLIANCES- } \\
\text { TOOLS }\end{array}$ & QUANTITY & PRICE \\
\hline Roller & 2 & $\begin{array}{c}\approx 15 €(\mathrm{per} \\
\text { item) }\end{array}$ \\
\hline Conveyor Belt & $148 \mathrm{~cm}$ & $\begin{array}{c}\approx 9 €(\mathrm{per} \\
\text { square } \\
\text { metre })\end{array}$ \\
\hline DC Motor & 1 & $\approx 50 €$ \\
\hline Laser Sensor & 1 & $\approx 807 €$ \\
\hline Thermal Camera & 1 & $\approx 17.000 €$ \\
\hline PLC & 1 & $\approx 580 €$ \\
\hline Photovoltaic Battery & 1 & $\approx 130 €$ \\
\hline
\end{tabular}

As we can see, it is not a cheap construction, especially for a single individual where he wants to experiment or study in detail the operation of the whole system. On the other hand, it is a pre-existing solution for an industry where it will not have the trouble to make an investment that can save time, less mistakes and better utilization for its employees. Also, a major part of the total cost is the energy saving offered by the photovoltaic module that will be installed.

In this chapter we will also look at future uses that this system might have. As we have said, this construction will be created to separate bottles that are filled from the ones that are not full filled. But this is not necessarily the only use.

Let's look at the construction in more detail. As we mentioned, the construction is divided into three parts, which is its supply, its base and its sensors. The part of the supply and the base of the construction do not give us anything else. What gives us great comfort in experimenting and finding other ideas is the sensors and the controller. As we know in the system we use a thermal camera and a laser where there are controlled by a
PLC. The laser sensor is suitable for distance calculations so we could have a standard distance of our product to ensure proper placement and no damage. We will also be able to measure the products they pass, that is, whenever the value of the distance of the sensor is reduced, it will be drawn that a product has been inserted. As for the heating system the options are many, as we know each object has a temperature so we can distinguish plastic from metal or even screws where they have been burned by the excessive use of the structure where they are located. However, especially in agricultural or food products, the camera is quite useful it can sort out two different types of fruit or the spoiled from the good. It can also separate objects of different color or glass from plastic bottles. All this is based on the controller we have chosen, where it gives us great ease in handling the thermo camera in the way we want it. Programming, however, is not an easy task, especially for such a sophisticated sensor as the camera, so the user must be knowledgeable in order to be able to fully manage the possibilities of the thermo-camera.

\section{ACKNOWLEDGMENTS}

All authors would like to thank the University of West Attica for the financial support provided to them for this research project.

\section{REFERENCES}

[1] WwW.wikipedia.org

[2] www.elergon.gr

[3] www.thermography.gr

[4] www.battery-expert.gr

[5] www.metadosi-ischios.gr http://www.metadosi-ischios.gr/article.php?ID=100

[6] ebooks.edu.gr http://ebooks.edu.gr/modules/ebook/show.php/DSGLC123/487/3182,12848/

[7] www.metadosi-ischios.gr http://www.metadosi-ischios.gr/article.php?ID=73

[8] www.infraspec.gr https://www.infraspec.gr/thermokameres-ke-chrisi-tous/

[9] www.flir.com www.flir.com/browse/industrial/fixed-thermal-cameras/

[10] www.eva-varouta-florou.gr by Eva Varouta Florou

[11] www.energeiakitechniki.gr http://www.energeiakitechniki.gr/?page_id=236

[12] www.selasenergy.gr http://www.selasenergy.gr/history.php

[13] www.schneider-electric.com 
https://www.schneider-

electric.com/en/product/TM241CE40R/controller-m241-

40-io-relay-ethernet/

[14] www.sick.com

https://www.sick.com/ag/en/distance-

sensors/displacement-measurement-sensors/dt20-

hi/c/g176377

[15] grobotronics.com

https://grobotronics.com/photo-resistor-ldr-5mm.html

[16] kmmb.gr

http://kmmb.gr/db/index.php?cPath=285

[17] www.solaire.gr

https://www.solaire.gr/autonoma/auto-calcs.html

[18] www.selasenergy.gr

http://www.selasenergy.gr/history.php

[19] grobotronics.com

https://grobotronics.com/premium-planetary-gear-motor-

313-rpm-hd.html

[20] www.zipy.gr

https://www.zipy.gr/p/ebay/forderbandrolle-

forderbandrollen-normrolle-tragrolle--89-mm-1-250-

$\mathrm{mm} / 282811740374 /$ 